\title{
The Higher the Price the Better the Result? The Placebo-Like Effects of Price and Brand on Consumer Judgments
}

\author{
I-Ling Ling ${ }^{1}$, Chih-Hui Shieh ${ }^{2}$, Jun-Fang Liao ${ }^{3}$ \\ ${ }^{1}$ Graduate Institute of Marketing \& Logistics/Transportation, National Chiayi University, Chiayi, Chinese Taipei \\ ${ }^{2}$ Department of Distribution Management, National Taichung University of Science \& Technology, \\ Taichung, Chinese Taipei \\ ${ }^{3}$ Department of International Business, Wenzao Ursuline College of Languages, Kaohsiung, Chinese Taipei \\ Email: chs101@nutc.edu.tw
}

Received May 16, 2012; revised June 17, 2012; accepted July 19, 2012

\begin{abstract}
This research investigates the placebo effects of price and brand on consumer judgment and focuses on how two psychological moderators, deliberation thinking and self-confidence, influence the placebo-like effects. Two experiments were conducted. The results show that although different prices and brands on the same product would not influence consumers' taste evaluation, they would affect participants' grades in the memory test. That means there is a placebo effect on price/brand cues. The results also indicate that the placebo effect of price is more influenced by the discounted price than the regular price when people have low deliberation thinking. Furthermore, the findings show that the effects of brand and self-confidence create an interaction effect. Self-confidence moderates the placebo effect of brand on consumers’ judgment. Theoretical and managerial implications of the findings are provided.
\end{abstract}

Keywords: Placebo Effect; Price; Brand; Deliberation Thinking; Self-Confidence

\section{Introduction}

Traditional models of economics presume that consumer utility for goods/services is determined by how much utility they will receive from a product, and then consumer accepts the transaction if the selling price is below his/her baseline price. The demand for a product is independent of the actual utility given by the product. Past research has shown that consumers sometimes use price information as a proxy for quality. In other words, price information may be used as a heuristic by which people infer the relative merit of a product or service [1]. Relevant to the current research, if price can increase expected value, then in the case of products it may be able to modify not merely perception but the actual product performance via the placebo effect.

A placebo has been defined by S. Stewart-Williams and J. Podd in medical literature as "a substance or procedure that has no inherent power to produce an effect that is sought or expected" [2]. A placebo I. Kirsch has observed placebo effect is essentially a "sugar pill." in numerous medical studies, from relatively benign maladies, such as warts and the common cold, to more serious diseases, such as diabetes, angina, and cancer [3]. Across multiple medical domains, the placebo effect has been investigated to be enduring and even capable of reversing the effects of active medications. Marketing factors, such as pricing and branding, are known to influence perception and expectation. Since expectation and beliefs are the main drivers of the placebo response, it is possible that marketing factors may modify the placebo response. Because the placebo response is an important component of many medical outcomes, it is logical to postulate that a manipulation of price or brand could have a direct effect on medical outcomes by modifying it.

In 2005, B. Shiv, Z. Carmon and D. Ariely (hereinafter SCA) [4] demonstrated that price was a salient piece of information because it affected behavior. In their article they document for the first time that nonconscious expectations about the relationship between quality and price can impact consumers in a placebo-like manner. Even when the price paid for goods or services has absolutely no relationship to its actual quality, consumers' nonconscious beliefs about the price-quality relationship change their actual experience with the product.

The purpose of this research is to extend SCA's findings and to investigate how two individual-difference 
factors, deliberation thinking and self-confidence, moderate the placebo-like effects of price and brand on consumers' judgment.

\section{Theoretical Background}

\subsection{Placebo Effect}

Placebo effects have long played a key role in medicine. For thousands of years many medical treatments were without specific objective effect and thus relied exclusively on placebo responses. The placebo effect is a topic of interest to psychologists and health practitioners in a wide variety of areas, and the question of the mechanisms underlying this effect is gaining increasing attention. Two mechanisms are believed to account for placebo effects: expectancy theory and classical conditioning. According to the expectancy theory, placebo effects arise because beliefs about a substance/procedure serving as a placebo activate expectations that a particular effect will occur, which then affect the subsequent effectiveness of the substance/procedure. The classical conditioning view considers consuming substances with known therapeutic effects to be conditioning trials [2].

In marketing, a placebo of this form might be a brand that claims to have certain properties that it does not actually possess and, through such claims, changes the consumer's behavior. In their work, SCA demonstrate that expectations play an important role in marketing placebo effects. Indeed, support for the efficacy of expectations goes back more than 1700 years: "He cures most in who most are confident," by M. P. Jensen and P. Karoly [5]. C. Irmak, L. G. Block, and G. J. Fitzsimons [6] applied SCA's [4] results by demonstrating the importance of motivation-a person's desire to experience the product's purported benefits-as a driver of marketing placebo effects. Motivation has also been shown to play a strong role in medical placebo studies such that when people want the physical symptoms, a placebo effect will more likely manifest [5,7].

\subsection{Price-Quality Assumption}

Since H. J. Leavitt [8] examined buyers' tendencies to use price as an indicator of quality, numerous studies have examined the price-quality relationship with little consensus as to its magnitude, generalizability, or statistical significance. A high price may be a handicap for a product's sales in the traditional model of economics. The fact that an expensive product is so pricy and still on the shelves may signal to consumers that it must warrant that price and must have some significant advantage over less expensive products. Higher prices may also serve as a signal. In many different areas of consumer products, no correlation has been found to exist, although quality and price move together more often in non-durable pro- ducts categories [9-11].

On the other hand, price is not the only important cue. The meta-analysis suggests that, for consumer products, the relationship between price and perceived quality and between brand name and perceived quality are positive and statistically significant [1]. Consumers may place greater value and quality on a product based on a placebolike effect. Price and brand are subjective ways to make an assessment of quality, because it is possible for consumers to bias some source of information based on individual difference factors.

\subsection{Individual Difference Factors}

\subsubsection{Deliberation Thinking}

Wilson and his colleagues [12] have shown that deliberation reduces satisfaction with personal judgments and attitude-behavior consistency and leads to less agreement with expert opinion about an evaluated object [13-15]. However, this counter intuitive finding has generally been thought to be a consequence of the limited processing capacity of deliberative thought by Ap. Dijksterhuis [16], Ap. Dijksterhuis and L. F. Nordgren [17]. Nordgren and Dijksterhuis [18] have found that deliberation reduces preference consistency. In their experiments 1 and 2, participants who deliberated on their preferences were less consistent in their evaluations compared to those who did not deliberate. Their experiment 3 demonstrated that this effect is due to the impediment of deliberation and not to the benefit of nondeliberation. Their also found that the extent to which deliberation decreases preference consistency depends upon the complexity of the information.

\subsubsection{Self-Confidence}

Consumer self-confidence is defined as the extent to which an individual feels capable and assured with respect to his or her marketplace decisions and behaviors. According to P. K. Adelman [19], consumer self-confidence reflects subjective evaluations of one's ability to generate positive experiences as a consumer in the marketplace. The behaviors of persons low in consumer selfconfidence are more subject to environmental circumstances and are more inclined to inconsistent decisionmaking than are those of persons who are high in consumer self-confidence [20].

J. E. Olsen, K. J. Thompson and T. K. Clarke [21] adapted consumer self-confidence scale in wine-related research. The impact of six distinct dimensions of consumer self-confidence on three different wine purchase situations is demonstrated. Results show the scale has the potential to inform both researchers and marketers about consumers' self-confidence related to wine purchases.

In this article, the authors extend and support SCA's findings by documenting for the first time a sugar pill 
placebo effects (price and brand) for everyday consumer products. Two studies were conducted in this research. Suggest writing this next sentence as two sentences. In study 1 , the authors propose that when participants eat the same chocolates priced differently, a placebo-like product (regular price vs discount price), will impact their short-term memory performance. However the levels of elaboration will moderate the placebo effect of price. In study 2, the authors find that the placebo effect manifests only for high confidence consumers who desire the arousing effects of a product. Expectations and the meaning of contextual effects drive the placebo response.

Four hypotheses were verified.

$H_{1}$ : People will perform greater in the regular price condition than in the discount price condition.

$\mathrm{H}_{2}$ : People with high elaboration will perform greater than people with low elaboration.

$H_{3 a}$ : People with low elaboration will perform a higher memory task in the regular price condition than in the discount price condition.

$H_{3 b}$ : People with high elaboration, there is no differrence of performance in memory task between regular price condition and the discount price condition.

$H_{4 a}$ : People with high self-confidence will perform a higher memory task in the high brand equity than in the middle brand equity and the low brand equity condition.

$H_{4 b}$ : People with high self-confidence will perform a higher memory task in the low brand equity than in the middle brand equity and the high brand equity condition.

\section{Research Strategy}

To examine both a difference in price as well as a difference in brand, on the efficacy of a placebo analgesic, two studies were conducted.

\subsection{Study I}

\subsubsection{Participants and Design}

One hundred and twenty undergraduate students in a computer lab were recruited for the experiment in exchange for a course credit. Participants were assigned to one of four conditions in a 2 (price: regular price vs discount price) $\times 2$ (elaboration: low vs high) betweensubjects design. The experimental stimulus was chocolate.

\subsubsection{Procedure and Measures}

Participants were led to a private cubicle that contained a personal computer and a prepared piece of chocolate. They were told that chocolate can improve people's short memory before the experiment. Participants were randomly assigned to one of two conditions: In the regular price condition, the price of a box of chocolate was tagged NT $\$ 1000^{1}$, Truffaut's. In the discount price condition, the price of a box of chocolate was tagged NT\$300 (70\% off of NT\$1000). The price was used as a manipulated variable. A regular price, NT\$1000 was tagged as a regular price, whereas the discount price condition provided $70 \%$ off discount (i.e., NT\$300). The measurement of elaboration process was asked for participants to write down the product knowledge of chocolate which they ate as a moderating variable in this experiment. An online memory test was conducted after participants ate the same chocolate tagged by different prices.

\subsubsection{Results}

Eight invalid samples were excluded because of wrong answer for the price. Elaboration was divided into two groups (median score). The means values for the performance of short memory task were shown in Figure 1. A 2 (price: regular vs $70 \%$ off discount) $\times 2$ (elaboration: high vs low) between-subjects ANOVA was performed. The result revealed a main effect of reference price $(F(1$, $109)=6.91, p<0.01$ ) on performance of short memory task. Memory performance was lower when the price was provided by $70 \%$ off $(M=14655.18, S D=5015.63)$ than when the price was regular $(M=16451.77, S D=$ 5033.45). $H_{1}$ was confirmed. There was a significant main effect of elaboration $(F(1,109)=8.33, p<0.01)$. Compared to low elaboration $(M=14503.57, S D=$ 5.37.39), the performance of short memory task was higher when participant's elaboration was high ( $M=$ 16603.39, $S D=4951.31)$. $H_{2}$ was verified.

More importantly, there is a marginal interaction effect of price and elaboration $(F(1,109)=2.82, p<0.1)$. Participants with low elaboration will perform for a higher memory performance in the regular price condition $(M=$ $16025.71, S D=4743.07)$ than in the discount price condition $(M=11966.67, S D=4552.51)$. People with high elaboration, there is no difference of memory perfor-

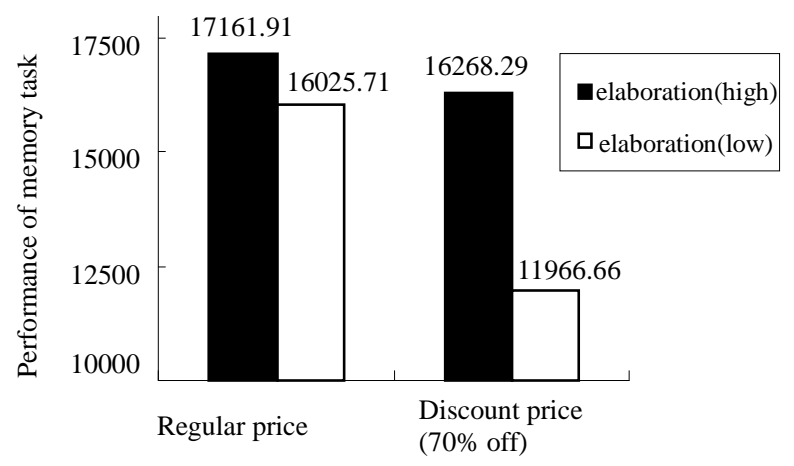

Figure 1. The performance of as a function of price and elaboration.

${ }^{1}$ NT\$: new Taiwan dollar. 
mance between regular price condition $(M=17161.90$, $S D=5530.05)$ and the discount price condition $(M=$ 16268.29, $S D=4621.69)$. $\mathrm{H}_{3 \mathrm{a}}$ and $\mathrm{H}_{3 \mathrm{~b}}$ were confirmed.

\subsection{Study II}

Brand names are cultural information and in essence a fundamental part of the ritual surrounding an object. Brands touch upon quality signaling, issues of personal identity and choice, nostalgia, and group affiliation. The purpose of experiment 2 was to test $H_{4 a}$ and $H_{4 b}$ whether self-confidence would have further impact on the placebo effect of brand.

\subsubsection{Participants and Design}

One hundred and eighty-eight students were recruited in the experiment in exchange for a lottery coupon. Participants were assigned to one of the experimental conditions in a 2 (brand equity: high vs medium vs low) $\times 2$ (self-condition: high vs low) between-subjects design. The experimental product was three kinds of brands.

\subsubsection{Procedure and Measures}

The procedure of experiment 2 was the same as experiment 1. Three brands, Truffaut's from France, 77 from Taiwan, and Meiji from Japan, were tagged as a placebolike brand. After tasting the manipulated chocolate, participants were asked to fill out the self-confidence scale by Bearden et al. [20]. According to self-confidence, the measurement of self-confidence was developed to identify people's aesthetic value by using a 20 -item on 5point scale.

\subsubsection{Results}

The manipulation check of brands showed a significant difference among conditions $\left(M_{\text {Truffettes }}=17413.56, M_{77}=\right.$ $\left.14544.62, M_{\text {Meiji }}=16742.19 ; F(2,182)=9.14, p<0.01\right)$. Post hoc tests showed that all means were different from each other at the 0.01 level. The difference reflected that the France brand Truffaut's was performed as having a higher brand equality than the 77 and the Meiji conditions.

An ANOVA with performance of memory task as dependent variable, and brand (Truffaut's vs 77 vs Meiji) and self-confidence (high vs low) as independent variables revealed a significant main effect of brand $(F(2$, $182)=9.14, p<0.01$ ), with the high brand equity leading to higher memory performance than the medium and low brand equity conditions $\left(M_{\text {Truffettest }}=17413.56, M_{77}=\right.$ 14544.62, $\left.M_{\text {Meiji }}=16742.19\right)$. The performance of memory task at the three different levels of brands indicated that the higher the brand equity, the greater the performance of memory task. The result was robust and demonstrated the experimental data are consistent with SCA (2005) and Irmak et al. (2005).
This effect is also qualified by a marginally significant interaction between brand and self-confidence on performance of memory task $(F(2,182)=0.24 p<0.1)$. The high and medium levels of brand equity increase the performance of memory task when self-confidence was high $\left(M_{\text {Truffettes }}=18440.00, M_{\text {Meiji }}=17291.43\right)$ compared to when self-confidence was low $\left(M_{\text {Truffettes }}=16658.82\right.$, $\left.M_{\text {Meiji }}=16079.31\right)$. But the effect of self-confidence decreased the performance of memory task when the selfconfidence was high (see Figure 2). In sum, self-confidence is a positively intrusive distraction that creates a sense of motivation for high brand equity but not for low brand equity. Thus $\mathrm{H}_{4}$ and $\mathrm{H}_{5}$ were confirmed.

\section{Discussion}

The results of this paper are consisting with SCA's study. The findings provide compelling evidence that marketing activities (e.g., price and brand) can lead to substantial placebo effects. As in SCA's research, the placebo effect that we observed led to changes in the participants' experience with the efficacy of the product. The placebo chocolate was capable of raising short memory, increaseing physical reflexes, enhancing mental alertness, and raising the self-reported arousal level for the participants. Notably, these placebo effects were only observed for highly motivated participants.

When participants reported a high degree of elaboration for the increased short memory task, the results showed that the placebo chocolate led to the same levels of performance. However, when participants had low levels of elaboration, consumption of the chocolate led to the same effects as a discount price. In addition to the important role that expectations plays in marketing placebo effects, these data suggest that motivation also plays an important role. It is possible in some situations that these two constructs interact in interesting ways. For example, in some situations, highly motivated people might change their expectations, thus leading to a placebo

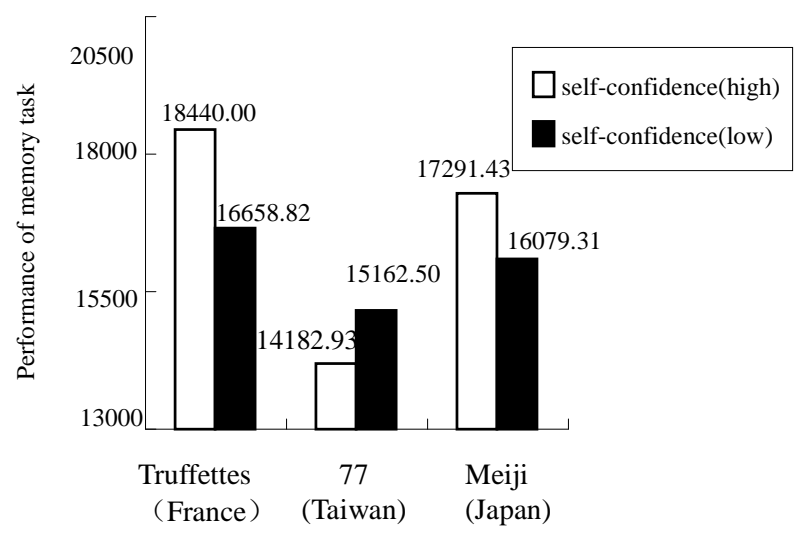

Figure 2. The performance of memory task as a function of brand and self-confidence. 
effect. However, our data do not support such a mediating role in the scenario we investigated. Our investigation suggests that sometimes consumers do not necessarily have to expect a product to work, they just have to want it to work $[4,6]$.

Although the medical literature reveals that the focus of investigations is on positive or beneficial effects of placebos, SCA clearly demonstrate that there can be substantial negative effects of placebos in marketing contexts. The physiological placebo effects we observe in this study also suggest that caution should be taken when making claims about products that seem harmless. An interesting aspect of SCA's work is the demonstration that marketing placebo effects can occur largely noncomsciously. Whereas the motivation that drove the placebo effect we observed in our study was conscious, it seems highly likely that the mechanism through which it operated may also have been nonconscious. Further research in this domain should prove extremely interesting both to marketers and to public policy makers.

\section{Acknowledgements}

This study was supported by a grant from the National Science Council (NSC 99-2410-H-415-022) for the first author. The authors gratefully acknowledge the reviewers in the 2012 Asia-Pacific Association for Consumer Research Conference for their insightful comments on earlier drafts of this paper.

\section{REFERENCES}

[1] A. R. Rao and K. B. Monroe, "The Effect of Price, Brand Name, and Store Name on Buyers' Perceptions of Product Quality: An Integrative Review,” Journal of Marketing Research, Vol. 26, No. 3, 1989, pp. 351-357. doi: $10.2307 / 3172907$

[2] S. Stewart-Williams and J. Podd, "The Placebo Effect: Dissolving the Expectancy versus Conditioning Debate," Psychological Bulletin, Vol. 130, No. 2, 2004, pp. 324340. doi:10.1037/0033-2909.130.2.324

[3] I. Kirsch, "Response Expectancy Theory and Application: A Decennial Review," Applied and Preventive Psychology, Vol. 6, No. 2, 1997, pp. 69-79. doi:10.1016/S0962-1849(05)80012-5

[4] B. Shiv, Z. Carmon and D. Ariely, "Placebo Effects of Marketing Actions: Consumers May Get What They Pay for,” Journal of Marketing Research, Vol. 42, No. 3, 2005, pp. 383-393. doi: 10.1509/jmkr.2005.42.4.383

[5] M. P. Jensen and P. Karoly, "Motivation and Expectancy Factors in Symptom Perception: A Laboratory Study of the Placebo Effect," Psychosomatic Medicine, Vol. 53, No. 2, 1991, pp. 144-152.

[6] C. Irmak, L. G. Block and G. J. Fitzsimons, "The Placebo Effect in Marketing: Sometimes: You Just Have to Want It to Work,” Journal of Marketing Research, Vol. 42, No.

\section{3, 2005, pp. 406-409. doi:10.1509/jmkr.2005.42.4.406}

[7] L. Vase, M. E. Robinson, G. N. Verne and D. D. Price, "The Contributions of Suggestion, Desire, and Expectation to Placebo Effects in Irritable Bowel Syndrome Patients: An Empirical Investigation,” Pain, Vol. 105, No. 1, 2003, pp. 17-25.

[8] H. J. Leavitt, "A Note on Some Experimental Findings about the Meaning of Price,” Journal of Business, Vol. 27, No. 3, 1954, pp. 205-210. doi:10.1086/294039

[9] P. C. Riesz, "Price-Quality Correlations for Packaged Food Products,” Journal of Consumer Affairs, Vol. 13, No. 2, 1979, pp. 236-247. doi:10.1111/j.1745-6606.1979.tb00142.x

[10] A. Wolinsky. "Prices as Signals of Quality," The Review of Economic Studies, Vol. 50, No. 4, 1983, pp. 647-658. doi: $10.2307 / 2297767$

[11] D. R. Lichtenstein, "The Relationship between Perceived and Objective Price-Quality," Journal of Marketing Research, Vol. 16, No. 4, 1989, pp. 429-443. doi:10.1086/294039

[12] T. D. Wilson, D. Lisle, J. W. Schooler, S. D. Hodges, K. J. Klaaren and S. J. LaFleur, "Introspecting about Reasons Can Reduce Post-Choice Satisfaction,” Personality and Social Psychology Bulletin, Vol. 19, No. 3, 1993, pp. 331339. doi:10.1177/0146167293193010

[13] J. B. Halberstadt and G. M. Levine, "Effects of Reasons Analysis on the Accuracy of Predicting Basketball Games,” Journal of Applied Social Psychology, Vol. 29, No. 3, 1999, pp. 517-530. doi:10.1111/j.1559-1816.1999.tb01399.x

[14] J. G. Johnson and M. Raab, "Take the First: OptionGeneration and Resulting Choices,” Organizational Behavior and Human Decision Processes, Vol. 91, No. 2, 2003, pp. 215-229. doi:10.1016/S0749-5978(03)00027-x

[15] T. D. Wilson and J. W. Schooler, “Thinking Too Much: Introspection Can Reduce the Quality of Preferences and Decisions,” Journal of Personality and Social Psychology, Vol. 60, No. 2, 1991, pp. 181-192. doi:10.1037/0022-3514.60.2.181

[16] Ap. Dijksterhuis, “Think Different: The Merits of Unconscious Thought in Preference Development and Decision Making," Journal of Personality and Social Psychology, Vol. 87, No. 5, 2004, pp. 586-598. doi:10.1037/0022-3514.87.5.586

[17] Ap. Dijksterhuis and L. F. Nordgren, “A Theory of Unconscious Thought," Perspectives on Psychological Science, Vol. 1, No. 2, 2006, pp. 95-109. doi:10.1111/j.1745-6916.2006.00007.x

[18] L. F. Nordgren and Ap. Dijksterhuis, "The Devil Is in the Deliberation: Thinking Too Much Reduces Preference Consistency," Journal of Consumer Research, Vol. 36, No. 1, 2009, pp. 39-46. doi:10.1086/596306

[19] P. K. Adelman, “Occupational Complexity, Control, and Personal Income: Their Relation to Psychological WellBeing in Men and Women,” Journal of Applied Psychology, Vol. 72, No. 4, 1987, pp. 529-537. doi:10.1037/0021-9010.72.4.529

[20] W. O. Bearden, D. M. Hardesty and R. L. Rose, “Con- 
sumer Self-Confidence: Refinements in Conceptualization and Measurement,” Journal of Consumer Research, Vol. 28, No. 3, 2001, pp. 121-134. doi:10.1086/321951

[21] J. E. Olsen, K. J. Thompson and T. K. Clarke, “Consumer
Self-Confidence in Wine Purchases,” International Journal of Wine Marketing, Vol. 15, No. 3, 2003, pp. 44-51. doi:10.1108/eb008762 